\title{
Open the gates
}

As we publish the first issue of Nature Reviews Psychology, we reflect on our ambitions to serve the entirety of psychological science and represent all psychological scientists.

$f f$ All research
areas and all
researchers -
across career
stages, geo-
graphy, and
identities - have
a home in our
journal

Psychology is a massive and sprawling field that crosses boundaries between the life sciences, clinical science and social science. Indeed, subfields of psychology often bear more resemblance to neighbouring fields than to each other. Yet psychology is united in its mission to understand the human mind and how it gives rise to human behaviour.

The scope of Nature Reviews Psychology reflects the full scope of the discipline: we are interested in timely syntheses spanning all subfields of cognitive, social and clinical psychology, including developmental aspects, methodological advances and more applied areas. The ethos of the Nature Reviews journals is to be authoritative and accessible. We believe this is especially important for a field as broad and diverse as psychology. That is, although our articles should be authoritative to topic specialists, they are written with an eye towards informing a broader audience. We aim to provide a one-stop shop where readers can find expert insights on recent developments in their own field as well as accessible overviews of research topics with which they are less familiar.

Psychologists seek to measure processes and constructs that are not directly observable, and they collect data from people who bring their idiosyncratic lifetimes of previous experiences - completely outside an experimenter's control - to the study. Moreover, the very questions psychologists ask do not emerge in a vacuum, but rather reflect the biases and backgrounds of the researchers. It should therefore be no surprise that rigorous debates about measurement, approaches, and interpretation are fundamental to the field. At the same time, different areas of psychology are subject to different methodological considerations. For example, approaches for studying reinforcement learning in the lab are vastly different from those needed to test how the principles of reinforcement learning can be leveraged to benefit individuals seeking to reduce substance use in their daily lives. Importantly, the enthusiasm for methodological innovation is evident across the entire discipline. We hope that featuring methods across our articles will foster appreciation for the richness of the psychologist's toolkit, and the fact that there is not a one-size-fits-all approach for studying psychology or improving methodological rigour.

A good review paper takes stock of where we are to point to where we need to go next. But review articles can do more than set a future research agenda (although this is an important ingredient in all our papers!). We seek to publish review articles that bring different areas of research together, reconcile competing theories or discrepant findings, promote methodological advances and link basic research to practical applications and implications for society. These aims are reflected in the Reviews and Perspectives in our first issue: Ullrich Ecker et al. review the literature on misinformation and interventions to combat it, a topic of acute societal relevance; Claudia von Bastian et al. seek to reconcile the disparate literature on cognitive training; Lauren Hadley et al. showcase the use of innovative methods to distinguish among theories of face-to-face social interaction; and Christopher Hopwood et al. bridge the literatures on personality and psychopathology and propose a research agenda to distinguish between them.

Our Review and Perspective articles, which integrate the empirical literature, are complemented by our Comment section, which offers a platform for thoughtful and compelling views on topics of relevance to the psychology community. For example, in this issue Stefan Gold and colleagues advocate for the use of platform trials to more efficiently test behavioural interventions, and David Gal and Derek Rucker argue that a bias towards experiments limits the potential of applied behavioural science. Importantly, we aim to publish Comments that go beyond documenting problems in research, meta-science, publishing or academia to offer practical solutions. This is exemplified by Comments from Jessica Schleider, who outlines specific actions that could promote service-centred research in clinical psychology, and Erica Wojcik, who provides concrete steps researchers can take to diversify their network of collaborators.

Of course, this is only our first issue, and so these articles are just a preview of the type of content we seek to publish and barely scratch the surface of the range of topics we endeavour to cover. We hope that each issue has something for everyone, and we aspire to be a venue that represents the full diversity of psychological science and is inclusive of all those who consider themselves psychological scientists. All research areas and all researchers across career stages, geography, and identities - have a home in our journal. Editors have a reputation for being gatekeepers. At Nature Reviews Psychology, we look forward to opening the gates. 